\title{
Transcriptome analysis reveals the tolerance mechanism of mantis shrimp (Oratosquilla oratoria) under lipopolysaccharide challenge
}

Daizhen Zhang ${ }^{1 \dagger}$, Peisong Zhao ${ }^{1,2 \dagger}$, Jun $\mathrm{Liu}^{3 \dagger}$, Tingting $\mathrm{Qi}^{1}$, Qiuning Liu ${ }^{1}$, Senhao Jiang ${ }^{1}$, Huabin Zhang ${ }^{1}$, Zhengfei Wang ${ }^{1}$, Boping Tang ${ }^{1}$, Ge Ding ${ }^{4}$ *

1.Jiangsu Key Laboratory for Bioresources of Saline Soils, Jiangsu Provincial Key Laboratory of Coastal Wetland Bioresources and Environmental Protection, Jiangsu Synthetic Innovation Center for Coastal Bio-agriculture, Yancheng Teachers University, Yancheng 224051, China; 2. College of Biotechnology and Pharmaceutical Engineering, Nanjing Tech University, Nanjing 211816,

China; 3. Key Laboratory of Biotechnology in Lianyungang Normal College, Lianyungang 222006, China; 4. Chemical and Biological Engineering College, Yancheng Institute of Technology, Yancheng 224003, China

* Correspondence authors:

Ge Ding (dinggeyc@163.com); Boping Tang (tangbp@yctu.edu.cn)

${ }^{\dagger}$ These authors contributed equally to this work. 
Table S1. Differentially expressed genes (DEGs) only expressed in OL or OP

\begin{tabular}{|c|c|c|c|c|c|c|c|}
\hline \multirow[b]{2}{*}{ Gene_id } & \multirow[b]{2}{*}{ OL } & \multirow[b]{2}{*}{ OP } & \multirow{2}{*}{$\begin{array}{l}\text { log2.Fold } \\
\text { change }\end{array}$} & \multirow[b]{2}{*}{ p.value } & \multirow{2}{*}{$\begin{array}{c}\text { q.value. } \\
\text { Storey.et. } \\
\text { al..2003 } \\
\end{array}$} & \multirow{2}{*}{$\begin{array}{c}\text { Gene } \\
\text { Length }\end{array}$} & \multirow[b]{2}{*}{ NR Description } \\
\hline & & & & & & & \\
\hline Cluster-42114.6145 & 73.17879 & 0 & 7.1934 & $2.38 \mathrm{E}-17$ & $6.26 \mathrm{E}-15$ & 4507 & $\begin{array}{l}\text { 1-phosphatidylinositol-4,5-bisph } \\
\text { osphate phosphodiesterase } \\
\text { delta-4 }\end{array}$ \\
\hline Cluster-42114.10146 & 70.54552 & 0 & 7.1405 & $7.87 \mathrm{E}-17$ & $1.99 \mathrm{E}-14$ & 1335 & -- \\
\hline Cluster-30781.1 & 70.37586 & 0 & 7.137 & $8.50 \mathrm{E}-17$ & $2.15 \mathrm{E}-14$ & 6693 & $\begin{array}{l}\text { hypothetical protein } \\
\text { DAPPUDRAFT_197668 }\end{array}$ \\
\hline Cluster-42114.7222 & 65.20627 & 0 & 7.0269 & $9.15 \mathrm{E}-16$ & $2.14 \mathrm{E}-13$ & 796 & -- \\
\hline Cluster-24557.1 & 59.26265 & 0 & 6.8891 & $1.47 \mathrm{E}-14$ & $3.15 \mathrm{E}-12$ & 2443 & $\begin{array}{l}\text { hypothetical protein } \\
\text { DAPPUDRAFT } 200500\end{array}$ \\
\hline Cluster-42114.9900 & 55.27209 & 0 & 6.7885 & $9.75 \mathrm{E}-14$ & $1.98 \mathrm{E}-11$ & 3728 & $\begin{array}{l}\text { Inositol-trisphosphate 3-kinase } \\
\text { A, partial }\end{array}$ \\
\hline Cluster-42114.11216 & 45.90709 & 0 & 6.5206 & $9.17 \mathrm{E}-12$ & $1.55 \mathrm{E}-09$ & 7078 & Talin-1 \\
\hline Cluster-42114.9398 & 45.19092 & 0 & 6.498 & $1.31 \mathrm{E}-11$ & 2.17E-09 & 5156 & hypothetical protein L798_12353 \\
\hline Cluster-60950.1 & 43.22769 & 0 & 6.4339 & $3.46 \mathrm{E}-11$ & 5.49E-09 & 3014 & $\begin{array}{l}\text { hypothetical protein } \\
\text { KGM_05776 }\end{array}$ \\
\hline Cluster-42114.9925 & 40.80552 & 0 & 6.3507 & $1.16 \mathrm{E}-10$ & $1.77 \mathrm{E}-08$ & 3458 & $\begin{array}{l}\text { Mitoferrin-2, partial } \\
\text { [Stegodyphus mimosarum }\end{array}$ \\
\hline Cluster-42114.8116 & 39.02134 & 0 & 6.2862 & $2.87 \mathrm{E}-10$ & 4.17E-08 & 5102 & $\begin{array}{l}\text { Macrophage mannose receptor } 1 \text {, } \\
\text { partial }\end{array}$ \\
\hline Cluster-42114.3435 & 35.4178 & 0 & 6.1464 & $1.81 \mathrm{E}-09$ & $2.40 \mathrm{E}-07$ & 2209 & -- \\
\hline Cluster-42114.5282 & 34.23486 & 0 & 6.0974 & 3.33E-09 & 4.28E-07 & 3356 & 796 \\
\hline Cluster-42114.7652 & 33.62658 & 0 & 6.0715 & 4.57E-09 & $5.82 \mathrm{E}-07$ & 2697 & $\begin{array}{l}\text { hypothetical protein } \\
\text { L798_05321, partial }\end{array}$ \\
\hline Cluster-62319.1 & 31.97062 & 0 & 5.9987 & $1.08 \mathrm{E}-08$ & $1.32 \mathrm{E}-06$ & 4729 & $\begin{array}{l}\text { hypothetical protein } \\
\text { DAPPUDRAFT_62579 }\end{array}$ \\
\hline Cluster-42114.10293 & 31.58829 & 0 & 5.9813 & 1.32E-08 & $1.59 \mathrm{E}-06$ & 1476 & $\begin{array}{l}\text { hypothetical protein } \\
\text { CAPTEDRAFT_225563 }\end{array}$ \\
\hline Cluster-42114.9393 & 28.92766 & 0 & 5.8544 & 5.39E-08 & $5.98 \mathrm{E}-06$ & 5739 & rab-3A-interacting protein-like \\
\hline Cluster-42114.9453 & 26.7205 & 0 & 5.7399 & $1.75 \mathrm{E}-07$ & $1.82 \mathrm{E}-05$ & 4141 & $\begin{array}{l}\text { phosphatidate phosphatase } \\
\text { LPIN3 isoform X4 }\end{array}$ \\
\hline Cluster-42114.6401 & 25.03014 & 0 & 5.6456 & 4.37E-07 & $4.28 \mathrm{E}-05$ & 547 & proteoliaisin \\
\hline Cluster-42114.11217 & 22.99968 & 0 & 5.5235 & $1.32 \mathrm{E}-06$ & $\begin{array}{r}0.0001222 \\
6 \\
\end{array}$ & 7033 & Talin-1 \\
\hline Cluster-42114.7197 & 22.56418 & 0 & 5.496 & $1.68 \mathrm{E}-06$ & $\begin{array}{r}0.0001534 \\
5 \\
\end{array}$ & 1814 & chitinase 4 precursor \\
\hline Cluster-42114.7300 & 22.4344 & 0 & 5.4876 & $1.81 \mathrm{E}-06$ & $\begin{array}{r}0.0001634 \\
3 \\
\end{array}$ & 2738 & $\begin{array}{l}\text { PREDICTED: BTB/POZ } \\
\text { domain-containing protein 1-like }\end{array}$ \\
\hline Cluster-42114.2893 & 22.37185 & 0 & 5.4836 & $1.87 \mathrm{E}-06$ & $\begin{array}{r}0.0001685 \\
5 \\
\end{array}$ & 2073 & $\begin{array}{l}\text { hypothetical protein } \\
\text { DAPPUDRAFT_203975 }\end{array}$ \\
\hline Cluster-42114.7111 & 22.02314 & 0 & 5.4609 & $2.27 \mathrm{E}-06$ & $\begin{array}{r}0.0002009 \\
2 \\
\end{array}$ & 2547 & hexokinase $\mathrm{A}$, isoform $\mathrm{B}$ \\
\hline Cluster-42114.9141 & 21.79719 & 0 & 5.4461 & $2.57 \mathrm{E}-06$ & $\begin{array}{r}0.0002249 \\
9 \\
\end{array}$ & 3824 & conserved hypothetical protein \\
\hline Cluster-42114.8542 & 21.45552 & 0 & 5.4233 & $3.10 \mathrm{E}-06$ & $\begin{array}{r}0.0002677 \\
8\end{array}$ & 3344 & $\begin{array}{l}\text { RecName: Full=Annulin; } \\
\text { AltName: } \\
\text { Full=Protein-glutamine } \\
\text { gamma-glutamyltransferase; } \\
\text { AltName: Full=Transglutaminase }\end{array}$ \\
\hline
\end{tabular}




\begin{tabular}{|c|c|c|c|c|c|c|c|}
\hline Cluster-42114.10778 & 21.39219 & 0 & 5.419 & $3.21 \mathrm{E}-06$ & $\begin{array}{r}0.0002760 \\
8\end{array}$ & 2308 & $\begin{array}{l}\text { vesicle-associated membrane } \\
\text { protein } 2\end{array}$ \\
\hline Cluster-36725.0 & 21.10994 & 0 & 5.3999 & $3.76 \mathrm{E}-06$ & $\begin{array}{r}0.0003192 \\
5\end{array}$ & 682 & -- \\
\hline Cluster-42114.5195 & 20.33826 & 0 & 5.3461 & $5.78 \mathrm{E}-06$ & 0.0004709 & 921 & $\begin{array}{l}\text { hypothetical protein } \\
\text { DAPPUDRAFT_315242 }\end{array}$ \\
\hline Cluster-66960.1 & 20.28353 & 0 & 5.3422 & $5.96 \mathrm{E}-06$ & 0.0004834 & 3741 & $\begin{array}{l}\text { sterile-alpha and armadillo motif } \\
\text { containing protein }\end{array}$ \\
\hline Cluster-42114.8166 & 19.79175 & 0 & 5.3068 & $7.84 \mathrm{E}-06$ & $\begin{array}{r}0.0006216 \\
7\end{array}$ & 5940 & $\begin{array}{l}\text { eukaryotic translation initiation } \\
\text { factor } 4 \text { gamma 1-like isoform } \\
\text { X3 }\end{array}$ \\
\hline Cluster-61423.1 & 19.75265 & 0 & 5.304 & $8.02 \mathrm{E}-06$ & $\begin{array}{r}0.0006327 \\
6\end{array}$ & 3318 & GD19599 \\
\hline Cluster-42114.9381 & 18.14126 & 0 & 5.1812 & $1.99 \mathrm{E}-05$ & 0.0014537 & 1847 & clathrin light chain \\
\hline Cluster-42114.8796 & 17.98724 & 0 & 5.1689 & $2.17 \mathrm{E}-05$ & 0.0015697 & 3435 & $\begin{array}{l}\text { heterogeneous nuclear } \\
\text { ribonucleoprotein } \mathrm{L} \text { isoform X1 }\end{array}$ \\
\hline Cluster-29010.1 & 16.84339 & 0 & 5.0741 & $4.16 \mathrm{E}-05$ & 0.0028488 & 2893 & -- \\
\hline Cluster-50757.0 & 16.72377 & 0 & 5.0638 & $4.46 \mathrm{E}-05$ & 0.0030191 & 1959 & -- \\
\hline Cluster-42114.4795 & 16.66591 & 0 & 5.0588 & 4.61E-05 & 0.0031078 & 3484 & $\begin{array}{l}\text { high affinity copper uptake } \\
\text { protein } 1 \text { isoform X1 }\end{array}$ \\
\hline Cluster-42114.3081 & 16.59007 & 0 & 5.0522 & $4.81 \mathrm{E}-05$ & 0.0032283 & 992 & Ras homolog enriched in brain \\
\hline Cluster-63068.4 & 16.48374 & 0 & 5.043 & $5.11 \mathrm{E}-05$ & 0.0034067 & 3473 & $\begin{array}{l}\text { hypothetical protein } \\
\text { DAPPUDRAFT_306979 }\end{array}$ \\
\hline Cluster-42114.15243 & 16.26091 & 0 & 5.0233 & $5.81 \mathrm{E}-05$ & 0.003807 & 5620 & $\begin{array}{l}\text { peptidase S24 family domain } \\
\text { containing protein }\end{array}$ \\
\hline Cluster-59076.0 & 16.0889 & 0 & 5.008 & $6.42 \mathrm{E}-05$ & 0.0041684 & 4082 & $\begin{array}{l}\text { PREDICTED: coatomer subunit } \\
\text { alpha }\end{array}$ \\
\hline Cluster-67660.0 & 16.06389 & 0 & 5.0057 & $6.51 \mathrm{E}-05$ & 0.0042191 & 3429 & $\begin{array}{l}\text { hypothetical protein } \\
\text { DAPPUDRAFT_308310 }\end{array}$ \\
\hline Cluster-42114.3638 & 15.9083 & 0 & 4.9917 & 7.12E-05 & 0.0045825 & 2510 & Selenoprotein S, partial \\
\hline Cluster-42114.10604 & 15.82855 & 0 & 4.9845 & $7.45 \mathrm{E}-05$ & 0.0047733 & 3930 & $\begin{array}{l}\text { StAR-related lipid transfer } \\
\text { protein }\end{array}$ \\
\hline Cluster-65845.1 & 15.74802 & 0 & 4.9771 & $7.81 \mathrm{E}-05$ & 0.0049745 & 4208 & -- \\
\hline Cluster-42114.2758 & 0 & 631 & -10.301 & \#\#\#\#\#\#\#\# & $1.18 \mathrm{E}-100$ & 5567 & $\begin{array}{l}\text { Phosphofructokinase family } \\
\text { protein }\end{array}$ \\
\hline Cluster-1526.2 & 0 & 14.5 & -4.8576 & $5.19 \mathrm{E}-05$ & 0.003453 & 2459 & -- \\
\hline Cluster-1792.0 & 0 & 17.31 & -5.1132 & $8.79 \mathrm{E}-06$ & $\begin{array}{r}0.0006887 \\
6\end{array}$ & 1760 & HipA domain protein, partial \\
\hline Cluster-23254.1 & 0 & 17.07 & -5.0936 & $1.02 \mathrm{E}-05$ & $\begin{array}{r}0.0007843 \\
8\end{array}$ & 630 & -- \\
\hline Cluster-23998.6 & 0 & 17.09 & -5.0948 & $1.01 \mathrm{E}-05$ & $\begin{array}{r}0.0007791 \\
5 \\
\end{array}$ & 1029 & -- \\
\hline Cluster-26628.1 & 0 & 19.81 & -5.3081 & $1.87 \mathrm{E}-06$ & $\begin{array}{r}0.0001684 \\
4\end{array}$ & 1209 & -- \\
\hline Cluster-31676.2 & 0 & 15.67 & -4.97 & $2.46 \mathrm{E}-05$ & 0.0017648 & 2223 & -- \\
\hline Cluster-42114.13580 & 0 & 103.1 & -7.6876 & $5.27 \mathrm{E}-25$ & $2.01 \mathrm{E}-22$ & 5674 & $\begin{array}{l}\text { elongation factor } \mathrm{G}, \\
\text { chloroplastic-like }\end{array}$ \\
\hline Cluster-37617.1 & 0 & 34.49 & -6.1081 & $3.47 \mathrm{E}-10$ & 4.99E-08 & 3421 & hypothetical protein \\
\hline Cluster-37621.2 & 0 & 26.39 & -5.7217 & $3.62 \mathrm{E}-08$ & $4.16 \mathrm{E}-06$ & 1880 & -- \\
\hline Cluster-42114.10367 & 0 & 28.4 & -5.8276 & $1.12 \mathrm{E}-08$ & $1.37 \mathrm{E}-06$ & 5136 & $\begin{array}{l}\text { dnaJ homolog subfamily B } \\
\text { member } 6 \text { isoform X2 }\end{array}$ \\
\hline Cluster-42114.10529 & 0 & 69.79 & -7.1249 & $3.39 \mathrm{E}-18$ & $9.39 \mathrm{E}-16$ & 1422 & transposase \\
\hline Cluster-42114.2760 & 0 & 95.27 & -7.574 & $1.86 \mathrm{E}-23$ & $6.72 \mathrm{E}-21$ & 5516 & $\begin{array}{l}\text { Phosphofructokinase family } \\
\text { protein }\end{array}$ \\
\hline Cluster-42114.11675 & 0 & 14.62 & -4.8698 & $4.80 \mathrm{E}-05$ & 0.0032248 & 1276 & C-type lectin-like protein \\
\hline
\end{tabular}




\begin{tabular}{|c|c|c|c|c|c|c|c|}
\hline Cluster-42114.11814 & 0 & 23.57 & -5.5586 & $1.92 \mathrm{E}-07$ & $1.98 \mathrm{E}-05$ & 997 & -- \\
\hline Cluster-42114.9988 & 0 & 48.7 & -6.6058 & $1.54 \mathrm{E}-13$ & $3.07 \mathrm{E}-11$ & 4487 & $\begin{array}{l}\text { 1-phosphatidylinositol-4,5-bisph } \\
\text { osphate phosphodiesterase } \\
\text { delta-4 }\end{array}$ \\
\hline Cluster-42114.13111 & 0 & 14.14 & -4.8216 & $6.53 \mathrm{E}-05$ & 0.0042286 & 2083 & $\begin{array}{l}\text { PREDICTED: heat shock } 70 \mathrm{kDa} \\
\text { protein, mitochondrial-like }\end{array}$ \\
\hline Cluster-42114.9589 & 0 & 39.75 & -6.313 & $1.88 \mathrm{E}-11$ & 3.07E-09 & 2668 & $\begin{array}{l}\text { hypothetical protein } \\
\text { L798_05321, partial }\end{array}$ \\
\hline Cluster-42114.13299 & 0 & 33.47 & -6.0647 & $6.18 \mathrm{E}-10$ & 8.63E-08 & 1621 & $\begin{array}{l}\text { PREDICTED: uncharacterized } \\
\text { protein LOC } 105897445\end{array}$ \\
\hline Cluster-42114.8236 & 0 & 33.87 & -6.0819 & 4.92E-10 & $6.96 \mathrm{E}-08$ & 1009 & myosin essential light chain \\
\hline Cluster-42114.12737 & 0 & 32.24 & -6.0108 & $1.24 \mathrm{E}-09$ & $1.67 \mathrm{E}-07$ & 5850 & chitin synthase \\
\hline Cluster-42114.7195 & 0 & 22.85 & -5.5144 & $2.95 \mathrm{E}-07$ & $2.96 \mathrm{E}-05$ & 1765 & chitinase 4 precursor \\
\hline Cluster-42114.14008 & 0 & 21.1 & -5.3992 & $8.49 \mathrm{E}-07$ & $8.07 \mathrm{E}-05$ & 1402 & kinesin-like protein KIF3A \\
\hline Cluster-42114.14344 & 0 & 16.79 & -5.0698 & $1.21 \mathrm{E}-05$ & $\begin{array}{r}0.0009205 \\
3\end{array}$ & 1930 & -- \\
\hline Cluster-42114.1993 & 0 & 17.82 & -5.1557 & $6.36 \mathrm{E}-06$ & $\begin{array}{r}0.0005135 \\
8 \\
\end{array}$ & 1663 & $\begin{array}{l}\text { lysosomal } \\
\text { alpha-glucosidase-like }\end{array}$ \\
\hline Cluster-50246.1 & 0 & 17.46 & -5.1259 & $7.99 \mathrm{E}-06$ & $\begin{array}{r}0.0006312 \\
4 \\
\end{array}$ & 1876 & $\begin{array}{l}\text { hypothetical protein } \\
\text { DAPPUDRAFT_230283 }\end{array}$ \\
\hline Cluster-42114.6976 & 0 & 15.58 & -4.9619 & $2.60 \mathrm{E}-05$ & 0.0018575 & 2173 & $\begin{array}{l}\text { transcription factor 7-like } 2 \\
\text { isoform X1 }\end{array}$ \\
\hline Cluster-904.0 & 0 & 14.43 & -4.8514 & $5.41 \mathrm{E}-05$ & 0.0035719 & 2338 & $\begin{array}{l}\text { adenine } \\
\text { phosphoribosyltransferase }\end{array}$ \\
\hline Cluster-42114.15466 & 0 & 19.03 & -5.2501 & $3.02 \mathrm{E}-06$ & $\begin{array}{r}0.0002605 \\
5 \\
\end{array}$ & 1634 & $\begin{array}{l}\text { hypothetical protein } \\
\text { PANDA_009509, partial }\end{array}$ \\
\hline Cluster-42114.1978 & 0 & 16.22 & -5.0194 & $1.74 \mathrm{E}-05$ & 0.0012833 & 592 & -- \\
\hline Cluster-42114.2440 & 0 & 41.76 & -6.3842 & $6.28 \mathrm{E}-12$ & $1.08 \mathrm{E}-09$ & 1843 & -- \\
\hline Cluster-42114.2983 & 0 & 41.96 & -6.3908 & $5.66 \mathrm{E}-12$ & $9.80 \mathrm{E}-10$ & 7168 & glycine-tRNA ligase \\
\hline Cluster-42114.3010 & 0 & 292.1 & -9.1903 & $2.32 \mathrm{E}-57$ & $1.87 \mathrm{E}-54$ & 4536 & glutathione reductase \\
\hline Cluster-42114.3063 & 0 & 18.93 & -5.2423 & $3.21 \mathrm{E}-06$ & $\begin{array}{r}0.0002760 \\
8\end{array}$ & 436 & -- \\
\hline Cluster-42114.3320 & 0 & 20.52 & -5.3586 & $1.21 \mathrm{E}-06$ & $\begin{array}{r}0.0001126 \\
9 \\
\end{array}$ & 685 & -- \\
\hline Cluster-42114.3437 & 0 & 35.2 & -6.1376 & $2.33 \mathrm{E}-10$ & $3.43 \mathrm{E}-08$ & 2190 & -- \\
\hline Cluster-42114.4449 & 0 & 21.33 & -5.4148 & $7.39 \mathrm{E}-07$ & $7.08 \mathrm{E}-05$ & 3327 & -- \\
\hline Cluster-42114.5938 & 0 & 18.52 & -5.2113 & $4.12 \mathrm{E}-06$ & $\begin{array}{r}0.0003483 \\
3\end{array}$ & 1702 & $\begin{array}{l}\text { Putative proline-rich protein } 21, \\
\text { partial }\end{array}$ \\
\hline Cluster-42114.6513 & 0 & 36.31 & -6.1821 & $1.26 \mathrm{E}-10$ & $1.90 \mathrm{E}-08$ & 859 & -- \\
\hline Cluster-42114.830 & 0 & 15.02 & -4.9091 & $3.71 \mathrm{E}-05$ & 0.0025628 & 1018 & -- \\
\hline Cluster-42114.8372 & 0 & 131 & -8.0335 & $2.39 \mathrm{E}-30$ & $1.07 \mathrm{E}-27$ & 5102 & $\begin{array}{l}\text { Macrophage mannose receptor } 1 \text {, } \\
\text { partial }\end{array}$ \\
\hline Cluster-42114.8565 & 0 & 19.7 & -5.3003 & $1.99 \mathrm{E}-06$ & $\begin{array}{r}0.0001785 \\
5 \\
\end{array}$ & 2659 & -- \\
\hline Cluster-42114.9821 & 0 & 21.33 & -5.415 & $7.37 \mathrm{E}-07$ & $7.08 \mathrm{E}-05$ & 4875 & -- \\
\hline Cluster-42114.9873 & 0 & 23.08 & -5.5286 & $2.57 \mathrm{E}-07$ & $2.60 \mathrm{E}-05$ & 2547 & -- \\
\hline Cluster-447.0 & 0 & 23.79 & -5.5721 & $1.69 \mathrm{E}-07$ & $1.76 \mathrm{E}-05$ & 2613 & $\begin{array}{l}\text { Toprim and HATPase } \mathrm{c} \text { and } \\
\text { DNA topoisoIV and DNA gy } \\
\text { raseA C and DNA gyraseB C and } \\
\text { DNA gyraseB domain containing } \\
\text { protein }\end{array}$ \\
\hline Cluster-67122.3 & 0 & 15.88 & -4.9892 & $2.15 \mathrm{E}-05$ & 0.0015602 & 1055 & -- \\
\hline
\end{tabular}


Table S2. 25 pathways with p-value less than 0.05 in functional enrichment analysis based on KEGG.

\begin{tabular}{|c|c|c|c|c|c|c|}
\hline Term & Database & ID & $\begin{array}{l}\text { Input } \\
\text { number }\end{array}$ & $\begin{array}{l}\text { Background } \\
\text { number }\end{array}$ & P-Value & $\begin{array}{l}\text { Corrected } \\
\text { P-Value }\end{array}$ \\
\hline Protein digestion and absorption & KEGG PATHWAY & ko04974 & 12 & 112 & $3.04 \mathrm{E}-07$ & $6.12 \mathrm{E}-05$ \\
\hline ECM-receptor interaction & KEGG PATHWAY & ko04512 & 10 & 117 & $1.85 \mathrm{E}-05$ & 0.001862 \\
\hline $\begin{array}{l}\text { Neuroactive ligand-receptor } \\
\text { interaction }\end{array}$ & KEGG PATHWAY & ko04080 & 6 & 42 & $6.88 \mathrm{E}-05$ & 0.004607 \\
\hline Focal adhesion & KEGG PATHWAY & ko04510 & 17 & 384 & 0.000103 & 0.005176 \\
\hline Amoebiasis & KEGG PATHWAY & ko05146 & 9 & 140 & 0.000363 & 0.014581 \\
\hline $\begin{array}{l}\text { Amino sugar and nucleotide } \\
\text { sugar metabolism }\end{array}$ & KEGG PATHWAY & ko00520 & 10 & 198 & 0.001042 & 0.034921 \\
\hline Riboflavin metabolism & KEGG PATHWAY & ko00740 & 3 & 16 & 0.002517 & 0.064393 \\
\hline Platelet activation & KEGG PATHWAY & ko04611 & 10 & 225 & 0.002563 & 0.064393 \\
\hline $\begin{array}{l}\text { AGE-RAGE signaling pathway } \\
\text { in diabetic complications }\end{array}$ & KEGG PATHWAY & ko04933 & 7 & 134 & 0.004804 & 0.107281 \\
\hline $\begin{array}{l}\text { Butirosin and neomycin } \\
\text { biosynthesis }\end{array}$ & KEGG PATHWAY & ko00524 & 2 & 7 & 0.007148 & 0.141071 \\
\hline $\begin{array}{l}\text { Glycosphingolipid biosynthesis } \\
\text { - globo series }\end{array}$ & KEGG PATHWAY & ko00603 & 3 & 25 & 0.00772 & 0.141071 \\
\hline Galactose metabolism & KEGG PATHWAY & ko00052 & 6 & 114 & 0.008495 & 0.142298 \\
\hline Pancreatic secretion & KEGG PATHWAY & ko04972 & 7 & 159 & 0.01133 & 0.167717 \\
\hline $\begin{array}{l}\text { Transcriptional misregulation in } \\
\text { cancer }\end{array}$ & KEGG PATHWAY & ko05202 & 7 & 160 & 0.011682 & 0.167717 \\
\hline Synaptic vesicle cycle & KEGG PATHWAY & ko04721 & 7 & 168 & 0.014784 & 0.198103 \\
\hline $\begin{array}{l}\text { Glycosphingolipid biosynthesis } \\
\text { - lacto and neolacto series }\end{array}$ & KEGG PATHWAY & ko00601 & 2 & 12 & 0.017219 & 0.216308 \\
\hline HIF-1 signaling pathway & KEGG PATHWAY & ko04066 & 8 & 233 & 0.02602 & 0.307648 \\
\hline Thyroid hormone synthesis & KEGG PATHWAY & ko04918 & 6 & 151 & 0.028176 & 0.314627 \\
\hline Arachidonic acid metabolism & KEGG PATHWAY & ko00590 & 5 & 114 & 0.03067 & 0.322643 \\
\hline MAPK signaling pathway & KEGG PATHWAY & ko04010 & 10 & 339 & 0.033603 & 0.322643 \\
\hline Endocytosis & KEGG PATHWAY & ko04144 & 13 & 487 & 0.033709 & 0.322643 \\
\hline Glycolysis / Gluconeogenesis & KEGG PATHWAY & ko00010 & 11 & 392 & 0.03603 & 0.329186 \\
\hline $\begin{array}{l}\text { Pathogenic Escherichia coli } \\
\text { infection }\end{array}$ & KEGG PATHWAY & ko05130 & 7 & 214 & 0.044438 & 0.380475 \\
\hline Lysosome & KEGG PATHWAY & ko04142 & 11 & 408 & 0.04543 & 0.380475 \\
\hline MicroRNAs in cancer & KEGG PATHWAY & ko05206 & 6 & 173 & 0.048242 & 0.387866 \\
\hline
\end{tabular}




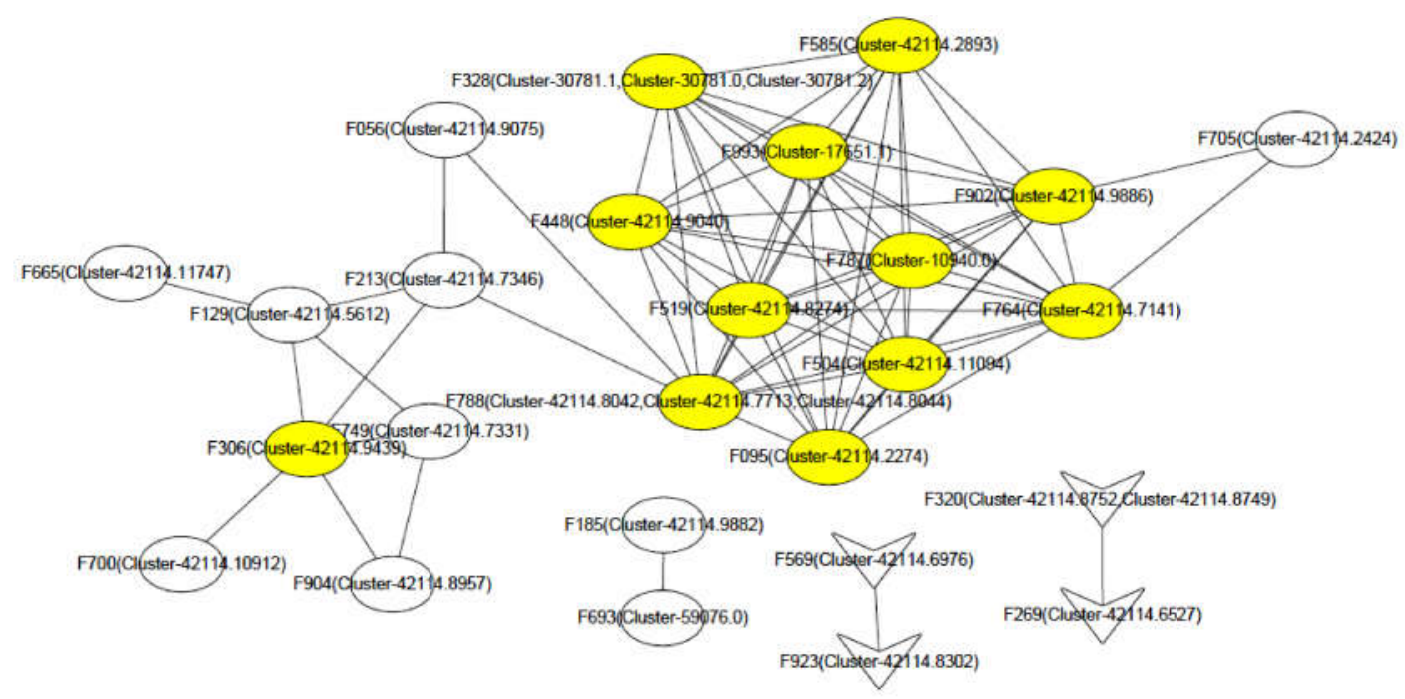

Figure S1. Protein-protein interaction of DEGs. Ellipse represents up-regulated DEGs, while "V" shape represents down-regulated DEGs. DEGs with higher "degree" $(\geq 5)$ were filled with yellow. 